\title{
PTSD: An After Effect of Covid-19
}

\author{
Dr. Vandana Gaur \\ Assistant Professor, Dept. of psychology \\ SDM Govt PG College Doiwala \\ Dehradun ,Uttarakhand \\ Email:vandanagaur97@gmail.com
}

\section{Abstract}

The covid-19 pandemic has many potential sources of trauma, such as experiencing the death of loved one. For some people, this can lead to post-traumatic stress disorder (PTSD). It can alsoexacerbate existing PTSD symptoms. This article will discuss how the covid-19 pandemic might lead to or worsen PTSD, as well as how to cope with the symptoms.

Post-traumatic stress disorder(PTSD) is a psychiatric disorder that is usually associated with people who have any prior experience of a traumatic event such as a natural disaster, a serious accident, war/combat, rape or other violent personal assault. As the intensity of psychological impact of covid-19 is increasing worldwide. PTSD affects as many as one in four people who experience traumatic events and contributes to marked functional disability, health impairment, and social dysfunction. The covid-19 pandemic has the potential to increase stress and anxiety, both because of the fear of infection and because of uncertainty about how the outbreak will affect people socially and economically.

Keywords: COVID-19;PTSD;wellness;DSM-IV

\author{
Reference to this paper \\ should be made as \\ follows: \\ Dr. Vandana Gaur \\ PTSD: An After \\ Effect of Covid-19 \\ Journal Global \\ Values, \\ Vol. XI, No.I \\ Article No.6 \\ pp. $43-50$ \\ http://anubooks.com/ \\ ?page_id $=6195$ \\ https://doi.org/ \\ 10.31995/ \\ jgv.2020.v11i01.006
}




\section{Introduction}

Psychological trauma is a common event involving threat to life, injury,or sexual violence, and it can result in a constellation of negative physical and psychological symptoms. Exposure to such traumatic events can lead to the development of acute stress disorder and finally PTSD if symptoms persist .PTSD is less common and develops when symptoms from a psychological trauma disrupt daily functioning and last for over a month.

Psychological trauma is common,with estimates of up to $60-85 \%$ of people having experienced a trauma with their lifetime. Post traumatic stress disorder(PTSD) is a condition that can develop after experiencing a psychological trauma when these reactions persist for a month or more cause substantial distress and disruption in one's life. PTSD is much less common than trauma exposure. An estimated $6 \%$ of men and $10 \%$ of women experience PTSD with their lifetime.

\section{Clinical Description of Post traumatic Stress Disorder}

Symptoms related to PTSD fall into three categories that include reliving the event, a sense of emotional numbness or depersonalization , and symptom of increased arousal(difficult sleeping, feeling irritated or easily angered, difficult concentrating). The diagnosis of PTSD occurs when a person has experienced symptoms for at least 1 month after a traumatic event, although symptoms may be delayed by several years.

The DSM-IV criteria of PTSD describes the setting event for PTSD as exposure to a traumatic event during which one feels fear, helplessness, or horror. Afterwards, victims reexperience the event through memories or nightmares. Sometimes these memories come on very suddenly and the victims find themselves reliving the whole event. When this occurs it is called a flashback. Victim also avoid anything that reminds them of the trauma and display a characteristic restriction or numbing of their emotional responsiveness, sometimes being unable to remember certain aspects of the event. The restriction or "numbing" of emotional experience seems to be a part of this avoidance and may be very disruptive to interpersonal relationships. It is possible that these victims are unconsciously attempting to avoid the experience of emotion itself, similarly to the behavior people with panic disorder, since experiencing intense emotions could bring back memories of the trauma. Finally, victims typically are chronically over aroused easily startled, and quick to anger. The DSM-IV criteria show that difficulty sleeping and recurring intrusive dreams of the event are prominent features of PTSD. 


\section{Trauma and the COVID pandemic}

COVID-19 has quickly become a global health concerns but also psychological concerns as people are exposed to unexpected deaths or threats of death. For example, healthcare workers who have close contact with COVID patients are not only exposed to the virus on a regular basis, but they may also be witnessing increased illnesses, deaths and supply shortage. In addition, patients admitted to the hospital with COVID-19 experience social isolation, physical discomfort, and fear for survival. These exposures increase the risk of PTSD.

It is important to note that the majority of people exposed to trauma recover within 30 days and do not develop PTSD. People who tested positive for covid-19 and survived after mandatory quarantine could develop long term mental health issues such as stress anxiety, and fear of the disease. Even if people are not directly affected by covid-19, the pandemic has been a significant stressor on people's lives across the globe. Considering covid-19's effects are global and long term, this psychological impact of covid-19 would be seen across many regions in the world. PTSD is already a significant healthcare issue that is often under diagnosed. Public heath agencies need to be able to address fall-out of the COVID-19 pandemic even after the infection is under control.

\section{Consequences of PTSD}

PTSD is often associated with profound changes in the autonomic nervous system, in particular an increase in activity of the sympathetic nervous system (the adrenaline system underlying the "fight, flight, or freeze" reactions)and a deficit in parasympathetic nervous system(the "rest and digest" system).PTSD is also associated with exaggerated activity in the brain networks associated with processing threat-detection and negative emotional responses; decreased activity in the networks involved in executive control, problem solving, and emotional regulation; and deficits in brain circuits involved in reward.

When it goes untreated, PTSD can last for decades. People with PTSD can be pernicious and insidious and can actually get worse rather than better over time. PTSD can be associated with substantial distress and disruption of social and occupational functioning, causing major problems in relationships and jobs.

\section{Predictors of PTSD: type and severity of trauma exposure}

It is important to note that the majority of peoples exposed to trauma recover within 30 days and do not develop PTSD. The type and severity of trauma exposure strongly predicts development of PTSD, with perpetrated interpersonal violence having much higher rates of PTSD than exposures like fires, natural disasters and any 
pandemic like covid-19.It is often under appreciated that medical events and procedures associated with life threat, even when they are successful, are associated with relatively high rates of PTSD development. It is vividly seen that prolonged treatment to COVID-19 patient has an immense effect on their mental health and has increased the severity of PTSD.

\section{PTSD can affect people differently}

The process begins with getting to know about any traumatic situation about , for or from a loved one. Usually the symptoms are quick to occur but in some cases it may take month or year.

These are four symptom types that characterize PTSD. These are:

- Having to experience the trauma all over again by mental conditions like nightmares or memory flashbacks.

- Avoiding stressors which were responsible for causing the situation that was responsible for trauma.

- Negative flow of emotions: The perception of individual about himself and the people associated with him changes drastically in a negative manner.

- Having to experience an aggravated form of arousal, insomnia(difficulty in sleeping), inability to concentrate and getting startled easily.

- If any of these symptoms persist for longer than 4 weeks, a person may diagnose PTSD.

\section{Eliminating the root cause of PTSD in a post-covid-19 world}

Its normal to feel a sense of hopelessness and loneliness in these times. But there are proven and protective ways for you to get through it.

- Be adaptable- a resilient behaviour is the most common kind of response noted in the case of trauma said Dr. Denise Sloan, a professor in psychiatry at Bostam University and associate director of the National Center for PTSD. Maximum amount of people are self sufficient to recover from PTSD.

- Purposefully increase your well-being-'If you are obsessed with this pandemic, you need to find ways to distract yourself', recommends Dr Edna Foa, a Professor in psychiatry at the university of Pennsylvania and one of the words leading experts in anxiety and trauma, We are in the middle of the trauma, not post stress," she said, highlighting that during a crisis we need to take breaks to soothe ourselves.

- n Stay connected and share your thoughts- one of the key resource which is particularly noted now is how we perceive social supports. We should always indulge in thinking that we are a part of the global community and are basically 
passing through a similar time frame altogether. Encourage yourself by watching videos and clips of people being thankful to health workers. Confide in others about what you are experiencing and ask others to share in return. Feeling connected protest against PTSD. "People need to communicate, especially if you are seeing horrible things." Dr Foa said.

- Be careful while narrating -Thought powerfully impact your feeling, so much so that you beliefs predict your risk of PTSD . One way to move away from negative thought, is to ask yourself, "Is this helpful?" self comparison is a meaningful way to cope with pain and while you're at it. Let go of judging others, studies show that doing so can significantly reduce loneliness.

- Too much thinking should be avoided - we are often stuck in a loop of thinking about abstract scenarios and the reasons which might cause them. This dosen't provide us with relief but instead, puts more pressure on our brain. To process thought about the pandemic that replay in your mind, one option is to put your concerns on paper, Expressive writing is one way to reflect without ruminating.

- Your credibility should be at your own stake -often times we feel that supressoing or hiding our emotions is the best way of handling them, but instead, we should take suitable considerations about them and treat them with kindness. Practicing mindfulness and viewing your experience with perspective helps you create a healthy relationship with your thoughts. Ultimately, its key to balance accepting difficult feelings, without multiplying them, while increasing your emotional immune system. Later, when this ends, if you find yourself becoming what Dr Foa described as a "super avoider"-some one no longer harmful- remember that its important to .

- Embrace your fears -The locus of control can be expended by prioritizing what matters the most to you. Cultivating a strong sense of purpose based on what you value, and using that to possibility, increasing both your physical strength and emotional recovery.

- Using techniques to stay calm-This might include anything from practicing yoga to eating well. For some, it might involve taking medication or avoiding stressors, such as watching the news focusing on a sense of control.

- Focusing on a sense of control-A person can also try to improve their sense of control and ability to endure stressful situations. For example, accepting circumstances that they are unable to change and focusing on what they can do may help reduce stress level.

- Remaining hopeful-A person can help themselves stay positive by: 
- 1- Maintaining a long-term perspective and looking to the future

- 2- Being patient and kind to themselves

- 3- Celebrating any successes

- 4- Taking breaks and doing enjoyable activities

\section{How to care for a person with PTSD}

To care for someone with PTSD, people can:

- Learn about it - understanding the effects of PTSD may help with understanding what that person is experiencing.

- Help manage their medication-A person can help support someone with PTSD by helping keep track of their medication.

- Listen-A person can let them know that they are there to listen, but they should not put pressure on them to talk about their symptoms.

- Encourage contact with other people-This may help the person build a support system.

If someone has PTSD, they may experience negative or suicidal thoughts. A person should not ignore any comments regarding self-harm, death, or wanting to die. If a person in crisis, anyone caring for them should contact a therapist or doctor.

\section{Tips for carers}

Looking after someone with PTSD can be stressful. It important for those looking after someone to also take care of themselves. If a person is looking after someone with PTSD ,they should:

- Not to feel guilty: It is normal to feel helpless and to not know all the answers.

- Take care of their mental health: A person should take time for themselves and do something they enjoy as after as possible.

- Take care of their physical health: Getting regular exercise may help a person cope with stress.

- Eat a healthful diet: This can help boost a person's energy levels.

\section{Treatment}

Treatment of PTSD can involve several forms of psychotherapy, including cognitive behavior therapy (CBT). CBT can help a person change unhelpful thinking patterns and behavior. It may also help them develop coping strategies.

Exposure therapy is one form of CBT. This can help people with PTSD by exposing them to the traumatic memories in a safe environment. Interpersonal therapy(IPT),present-centered therapy(PCT), and mindfulness-based therapies is also very important to treat PTSD. 
It is usually difficult to seek a proper kind of psychiatric help in virtue of this current pandemic. However, people suffering with PTSD can take help from remedies like tele psychiatry.

\section{Conclusion}

During this difficult time of COVID-19 pandemic there are many attributes which may cause the situation to get worsen. Also, physical distancing and other lockdown measures can create challenges for treatment in some people. Some people with PTSD may benefit from trying self help approaches, Having sources of support is also important. Other people may require professional help or medication. Global Data expects that the Covid-19 pandemic may cause even higher prevalence of PTSD in the severely affected areas where the population is at high risk of infection and where significant changes to daily living had occurred due to pandemic response. Considering Covid-19's effects are global and long -term, this psychological impact of Covid-19would be seen across many regions in the world. Public health agencies need to e able to address fall-out of the Covid-19 pandemic even after the infection is under control.

\section{References}

1 Find help(n.d) https://adaa.org/finding-help

2 Foa E.B.,2008, P araxetine CR augmentation for posttraumatic sress disorder refractory to prolonged exposure therapy. Journal of Clinical Psychiatry 69(3):400-405.

3 Foa E.B.,2008, Anger and posttraumatic stress disorder symptoms in crime victims: A Longitudinal Analysis. Journal of Consulting and Clinical Psychology 76(2):208-218.

4 Greenberg, N., et al.(2020).Managing mental health challenges faced by healthcare workers during COVID-19 pandemic.

5 Haghnia, Y., et al.(2019). Telepsychiatry-based case for the treatment followup of iranian war veterans with post-traumatic stress diorder: A randomized controlled trial.

6 https:www.ncbi.nlm.nih.gov/pmc/articles/PMC6661519/

7 Helping a family member who has PTSD.(n.d)

$\underline{8}$ http://www.ptsd.va.gov/family/how family member.asp

9 Horesh, D.,\& Brown,A.D.(2020).Traumatic stress in the age of COVID19: A call to close critical gaps and adapt to new realities.

10 https://psynet.apa.org/fulltext/2020-25108.001.html 
11 Managing stress associated with the COVID-19 virus outbreak(n.d)

12 https://www.ptsd.va.gov/COVID managing stress.asp

13 Post-traumatic stress disorder.(n.d.)

14 htttps://www.nimh.nih.gov/health/publication/post-traumatic-stress -disorderptsd/index.shtml PTSD basics.n.d.)

$\underline{16}$ https://www.ptsd.va.gov/understand/what/ptsd basics.asp

17 Self help and coping.9n.d.)

18 https://www.ptsd.va.gov/gethelp/selfhelp coping.asp

19 Sloan DM.(2019), Brief novel therapies for PTSD: Written Exposure Therapy.Curr Treat Options Psychiatry. :6(2):99-106.PMID:31245252.

20 Symptoms of PTSD.(n.d)

21 https:adaa.org/understanding:anxiety/posttraumatic -stress-disorder-ptsd/ symptoms

22 Treatment for PTSD.(2020)

23 https://adaa.org/understanding:anxiety/posttraumatic-stress-disorder-ptsd/ treatment 